\title{
SPECTRAL ESTIMATES FOR A NONHOMOGENEOUS DIFFERENCE PROBLEM
}

\author{
ALEXANDRU KRISTÁLY ${ }^{*, \dagger, \|, ~ M I H A I ~ M I H A ̆ I L E S C U ~}{ }^{\dagger, \ddagger, * *}$, \\ VICENŢIU RĂDULESCU ${ }^{\ddagger}, \S, \dagger \dagger$ and STEPAN TERSIAN $₫$, \\ *Department of Economics, University of Babeş-Bolyai \\ 400591 Cluj-Napoca, Romania \\ ${ }^{\dagger}$ Department of Mathematics, Central European University \\ 1051 Budapest, Hungary \\ ${ }^{\ddagger}$ Department of Mathematics, University of Craiova \\ 200585 Craiova, Romania \\ §Institute of Mathematics "Simion Stoilow" of the Romanian Academy \\ P.O. Box 1-764, 014700 Bucharest, Romania \\ "Department of Mathematical Analysis \\ University of Rousse, 7017 Rousse, Bulgaria \\ " alexandrukristaly@yahoo.com \\ **mmihailes@yahoo.com \\ ††vicentiu.radulescu@imar.ro \\ ¥†sterzian@ru.acad.bg
}

Received 4 June 2009

Dedicated to Professor Luis Sanchez on the occasion of his 60th birthday

\begin{abstract}
We study an eigenvalue problem in the framework of difference equations. We show that there exist two positive constants $\lambda_{0}$ and $\lambda_{1}$ verifying $\lambda_{0} \leq \lambda_{1}$ such that any $\lambda \in\left(0, \lambda_{0}\right)$ is not an eigenvalue of the problem, while any $\lambda \in\left[\lambda_{1}, \infty\right)$ is an eigenvalue of the problem. Some estimates for $\lambda_{0}$ and $\lambda_{1}$ are also given.
\end{abstract}

Keywords: Eigenvalue problem; discrete boundary value problem; critical point; continuous spectrum.

Mathematics Subject Classification 2010: 47A75, 35B38, 35P30, 34L05, 34L30

\section{Introduction and Main Results}

Discrete boundary value problems have been intensively studied in the last decade. The modeling of certain nonlinear problems from biological neural networks, economics, optimal control and other areas of study have led to the rapid development of the theory of difference equations (see the monographs of $[1,9]$ and the papers of $[2,3,6,7,12,14,15]$ and the reference therein).

${ }^{\dagger}$ Corresponding author. 
In view of developing a viable theory of discrete boundary problems, special attention has been given to the study of the spectrum of certain eigenvalue problems. A classical result in the theory of eigenvalue problems involving difference equations asserts that the spectrum of the problem

$$
\left\{\begin{array}{l}
-\Delta(\Delta u(k-1))=\lambda u(k), \quad k \in[1, T] \\
u(0)=u(T+1)=0
\end{array}\right.
$$

where $T \geq 2$ is an integer, $[1, T]$ is the discrete interval $\{1,2, \ldots, T\}$ and $\Delta u(k)=$ $u(k+1)-u(k)$ is the forward difference operator, is finite and all the eigenvalues are positive. Since $u \mapsto \Delta(\Delta u(\cdot))$ is a linear operator, the above statement is well illustrated if one considers, instead of (1) the algebraic equation $c s=\lambda s, s \in \mathbb{R}$, where $c>0$ is a fixed number.

On the other hand, some recent advances obtained in $[2,6,12]$ show that for some eigenvalue problems involving difference operators the spectrum contains a continuous family of eigenvalues.

The goal of this paper is to complete the studies begun in the above papers by presenting a new phenomenon concerning the behavior of eigenvalues of a nonhomogeneous difference equation. Using the above notations, this paper is concerned with the study of the eigenvalue problem

$$
\left\{\begin{array}{l}
-\Delta(\Delta u(k-1))+|u(k)|^{q-2} u(k)=\lambda g(k)|u(k)|^{r-2} u(k), \quad k \in[1, T], \\
u(0)=u(T+1)=0
\end{array}\right.
$$

where $q$ and $r$ are two real numbers satisfying $2<r<q$ and $g:[1, T] \rightarrow(0, \infty)$ is a given function.

Similar to the previous case, we formally consider instead of (2) the algebraic equation

$$
c s+|s|^{q-2} s=\lambda|s|^{r-2} s, \quad s \in \mathbb{R} .
$$

Note that if we define $f:(0, \infty) \rightarrow(0, \infty)$ by $f(t)=c t^{2-r}+t^{q-r}$ and the positive number $t_{0}=\left(\frac{c(r-2)}{q-r}\right)^{\frac{1}{q-2}}$, Eq. $\left(2^{\prime}\right)$ has no nonzero solutions for $0<\lambda<f\left(t_{0}\right)$, while for any $\lambda \geq f\left(t_{0}\right)$, Eq. $\left(2^{\prime}\right)$ has nonzero solutions.

By finding solutions to problem (2), we expect to obtain a similar phenomenon as the one we described for the algebraic equation $\left(2^{\prime}\right)$. Note, however, that the presence of the function $g$ as well as the nonhomogeneous nature of problem (2) make this problem more difficult. We shall prove the existence of two positive numbers $\lambda_{0}$ and $\lambda_{1}$, with $\lambda_{0} \leq \lambda_{1}$ such that for $\lambda \in\left(0, \lambda_{0}\right)$ problem (2) has no nonzero solutions while for any $\lambda \in\left[\lambda_{1}, \infty\right)$ problem (2) has nonzero solutions in a specific function space. Moreover, useful estimates will be also given for $\lambda_{0}$ and $\lambda_{1}$ with respect to the initial data $q, r, T$ and $g$.

In order to describe our result in its full generality we first define the function space

$$
H=\{u:[0, T+1] \rightarrow \mathbb{R} ; u(0)=u(T+1)=0\}
$$


Clearly, $H$ is a $T$-dimensional Hilbert space (see [2]) with the inner product

$$
(u, v)=\sum_{k=1}^{T+1} \Delta u(k-1) \Delta v(k-1), \quad \forall u, v \in H .
$$

The associated norm is defined by

$$
\|u\|=\left(\sum_{k=1}^{T+1}|\Delta u(k-1)|^{2}\right)^{1 / 2} .
$$

We say that $\lambda \in \mathbb{R}$ is an eigenvalue of problem (2) if there exists $u \in H \backslash\{0\}$ such that

$$
\begin{aligned}
& \sum_{k=1}^{T+1} \Delta u(k-1) \Delta v(k-1)+\sum_{k=1}^{T}|u(k)|^{q-2} u(k) v(k) \\
& \quad-\lambda \sum_{k=1}^{T} g(k)|u(k)|^{r-2} u(k) v(k)=0, \quad \forall v \in H .
\end{aligned}
$$

The function $u$ in the above definition will be called an eigenvector of problem (2). The set of all eigenvalues of problem (2) will be called the spectrum of problem (2).

The following theorem represents the main result of our paper.

Theorem 1. Let $2<r<q, T \geq 2$ and $g:[1, T] \rightarrow(0, \infty)$ be a given function. Then there exist two positive constants $\lambda_{0}$ and $\lambda_{1}$ with $\lambda_{0} \leq \lambda_{1}$ such that no $\lambda \in\left(0, \lambda_{0}\right)$ is an eigenvalue of problem (2) while any $\lambda \in\left[\lambda_{1}, \infty\right)$ is an eigenvalue of problem (2). Moreover, we have

$$
\lambda_{1} \leq \frac{r}{2} \lambda_{0} \quad \text { and } \quad \frac{4}{(T+1)^{2}|g|_{\infty}} \leq \lambda_{0} \leq \lambda_{1} \leq \frac{r(q-2)}{(q-r) \sum_{k=1}^{T} g(k)}\left(\frac{T(q-r)}{q(r-2)}\right)^{\frac{r-2}{q-2}}
$$

where $|g|_{\infty}=\max _{k \in[1, T]} g(k)$.

Notation. For any $a$ and $b$ integers satisfying $a<b$ we denote by $[a, b]$ the discrete interval $\{a, a+1, \ldots, b\}$.

\section{Some Estimates of Eigenvalues}

In this section, we will point out certain remarks on how we can estimate the positive eigenvalues corresponding to positive eigenvectors for the problem

$$
\left\{\begin{array}{l}
-\Delta(\Delta u(k-1))=\lambda u(k), \quad k \in[1, T], \\
u(0)=u(T+1)=0 .
\end{array}\right.
$$


In this section, the main result is given by Theorem 2 which is of interest in its own right as well:

Theorem 2. Let $\lambda>0$ be an eigenvalue of problem (4) with the property that the corresponding eigenvector $u=\{u(k): k \in[0, T+1]\}$ is positive, i.e. $u(k)>0$ for any $k \in[1, T]$. Then we have the estimates

$$
\frac{4}{(T+1)^{2}}<\lambda \leq \min \left\{1, \frac{1}{T} \cdot\left(1+\frac{\max \{u(1), u(T)\}}{\min \{u(1), u(T)\}}\right)\right\} .
$$

Proof. First, we point out certain general remarks on the behavior of $\Delta u(k)$ for $k \in[0, T]$. Since $u(k)>0$ for $k \in[1, T]$ satisfies Eq. (2) and $\lambda>0$ we have

$$
\Delta(\Delta u(k-1))=-\lambda u(k)<0, \quad \forall k \in[1, T] .
$$

Thus, we deduce that the sequence $(\Delta u(k))$ is decreasing for $k \in[0, T]$.

Second, we show that the left inequality holds true. In order to do that, we start by defining

$$
m=\max \{s \in[1, T] ; \Delta u(s-1) \geq 0, \Delta u(s)<0\} .
$$

Undoubtedly, $m$ can be defined as the above since we have $u(T+1)=0$ and $\Delta u(T)=u(T+1)-u(T)=-u(T)<0$. (Actually, $m$ is the largest local maximum point of $u$ in $[1, T]$.)

On the other hand, since $\Delta u(m)<0$ and $(\Delta u(k))$ is a decreasing sequence for $k \in[0, T]$ we notice that

$$
\Delta u(k)<0, \quad \forall k \in[m, T]
$$

and thus,

$$
u(k+1)<u(k), \quad \forall k \in[m, T],
$$

i.e. the sequence $(u(k))$ is strictly decreasing for $k \in[m, T]$. A similar argument, based on the fact that $\Delta u(m-1) \geq 0$ implies that $\Delta u(k) \geq 0$ for any $k \in[0, m-1]$, i.e. the sequence $(u(k))$ is nondecreasing for $k \in[0, m]$.

Adding the identities $u(k)-u(k-1)=\Delta u(k-1)$ for $k \in[m+1, T+1]$ we obtain $u(T+1)-u(m)=\sum_{k=m+1}^{T+1} \Delta u(k-1) \geq(T+1-m) \Delta u(T)$, i.e.

$$
\frac{-u(m)}{T+1-m} \geq \Delta u(T) \text {. }
$$

Since by Eq. (4) we have that

$$
\Delta(\Delta u(k-1))=-\lambda u(k), \quad \forall k \in[m, T],
$$

summing the above relations with respect to $k \in[m, T]$ we obtain

$$
\Delta u(T)-\Delta u(m-1)=-\lambda \sum_{i=m}^{T} u(i)
$$


Taking into account that $\Delta u(m-1) \geq 0$ the above equality implies

$$
\Delta u(T) \geq-\lambda \sum_{i=m}^{T} u(i)
$$

The above inequality, relation $(6)$ and the fact that the sequence $(u(k))$ is decreasing for $k \in[m, T]$ yield

$$
\frac{-u(m)}{T+1-m} \geq-\lambda \sum_{i=m}^{T} u(i) \geq-\lambda u(m) \sum_{i=m}^{T} 1
$$

or

$$
\lambda \sum_{i=m}^{T} 1 \geq \frac{1}{T+1-m}
$$

In order to go further, we add the identities $u(k)-u(k-1)=\Delta u(k-1)$ for $k \in[1, m]$, obtaining that $u(m)-u(0)=\sum_{k=1}^{m} \Delta u(k-1)$. This inequality and the fact that the sequence $(\Delta u(k))$ is decreasing for $k \in[0, T]$ imply

$$
\Delta u(0) \geq \frac{u(m)}{m}
$$

Since by Eq. (4) we have that

$$
\Delta(\Delta u(k-1))=-\lambda u(k), \quad \forall k \in[1, m],
$$

summing the above relations with respect to $k \in[1, m]$ we obtain

$$
\Delta u(m)-\Delta u(0)=-\lambda \sum_{i=1}^{m} u(i) .
$$

But $\Delta u(m)<0$ and taking into account that relation (8) holds true, we infer by the above equality

$$
\frac{u(m)}{m}<\lambda \sum_{i=1}^{m} u(i) .
$$

Using the fact that $(u(k))$ is nondecreasing for $k \in[0, m]$ we find

$$
\frac{1}{m}<\lambda \sum_{i=1}^{m} 1 \text {. }
$$

Now, by (7) and (9), we get

$$
\lambda \sum_{i=m}^{T} 1+\lambda \sum_{i=1}^{m} 1>\frac{1}{T+1-m}+\frac{1}{m} .
$$

Thus, we conclude that

or

$$
\lambda(T+1)>\frac{4}{T+1},
$$

$$
\lambda>\frac{4}{(T+1)^{2}} .
$$


Finally, we prove the second inequality. By Eq. (4) we have

$$
\Delta u(k)-\Delta u(k-1)=-\lambda u(k), \quad \forall k \in[1, T] .
$$

Summing the above relations, we find

$$
u(T)+u(1)=\lambda \sum_{i=1}^{T} u(i)
$$

Since $u(k)>0$ by using the above relation, we find, on the one hand, that

$$
u(T)+u(1) \geq \lambda T \min _{k \in[1, T]} u(k),
$$

or

$$
\frac{u(T)+u(1)}{T \min _{k \in[1, T]} u(k)} \geq \lambda
$$

and on the other hand,

$$
u(T)+u(1) \geq \lambda(u(1)+u(T))
$$

or

$$
1 \geq \lambda
$$

Furthermore, we notice that if $u\left(k_{0}\right)=\min _{k \in[1, T]} u(k)$ then $k_{0} \in\{1, T\}$. Indeed, let us assume by contradiction that $k_{0} \in[1, T] \backslash\{1, T\}$. Then, since

$$
\Delta u\left(k_{0}\right)-\Delta u\left(k_{0}-1\right)=-\lambda u\left(k_{0}\right),
$$

or

$$
0 \leq u\left(k_{0}+1\right)-2 u\left(k_{0}\right)+u\left(k_{0}-1\right)=-\lambda u\left(k_{0}\right)<0,
$$

we obtain a contradiction. Consequently, $k_{0} \in\{1, T\}$. That fact and relation (10) yield

$$
\frac{1}{T} \cdot\left(1+\frac{\max \{u(1), u(T)\}}{\min \{u(1), u(T)\}}\right) \geq \lambda .
$$

Theorem 2 is completely proved.

Remark 1. We emphasize that for the estimate in the left-hand side of (5) we can give an alternative proof. This idea is described in what follows. The eigenvalues of problem (4) can be calculated directly, solving the linear second-order difference equation

$$
\Delta(\Delta u(k-1))+\lambda u(k)=0,
$$

(see, e.g. [9, Chap. 3], [4, p. 38]). The eigenvalues of (4) are

$$
\lambda_{k}=2\left(1-\cos \left(\frac{k \pi}{T+1}\right)\right)=4 \sin ^{2}\left(\frac{k \pi}{2(T+1)}\right), \quad k \in[1, T],
$$

and the corresponding eigenvectors are

$$
\varphi_{k}=\left\{0, \sin \left(\frac{k \pi}{T+1}\right), \sin \left(\frac{2 k \pi}{T+1},\right), \ldots, \sin \left(\frac{T k \pi}{T+1},\right), 0\right\} .
$$


Note that $0<\lambda_{k}<4$ and the estimate from the left-hand side in (5) implies

$$
m(T):=\frac{4}{(T+1)^{2}}<\lambda_{1}=4 \sin ^{2}\left(\frac{\pi}{2(T+1)}\right),
$$

or, equivalently

$$
\frac{1}{(T+1)}<\sin \left(\frac{\pi}{2(T+1)}\right) .
$$

That fact follows also from the elementary inequality

$$
x<\sin \left(\frac{\pi x}{2}\right), \quad \forall x \in(0,1) .
$$

The last inequality is equivalent with the following fact

$$
\frac{2}{\pi} x<\sin (x), \quad \forall x \in\left(0, \frac{\pi}{2}\right),
$$

which geometrically means that the graph of $\sin (x)$ is above the chord which joints the points $(0,0)$ and $(\pi / 2,1)$.

Since problem (4) is homogeneous, we can assume that $u(1)=1$. Then the right-hand side estimate of (5) can be replaced by

$$
M(u, T):=\min \left\{1, \frac{1+u(T)}{T u(T)}, \frac{1+u(T)}{T}\right\},
$$

and the corresponding eigenvector of $\lambda_{k}$ can be chosen as

$$
u_{k}=\left\{0,1, \frac{\sin (2 k \pi /(T+1))}{\sin (k \pi /(T+1))}, \ldots, \frac{\sin (T k \pi /(T+1))}{\sin (k \pi /(T+1))}, 0\right\} .
$$

Note that $\sin (T \pi /(T+1))=\sin (\pi /(T+1))$ which implies that $M\left(u_{1}, T\right)=2 / T$ and

$$
\frac{4}{(T+1)^{2}}<\lambda_{1}<\frac{2}{T}
$$

We also have $\sin (2 T \pi /(T+1))=-\sin (2 \pi /(T+1))$, which implies that $u_{2}(T)=-1$ and $M\left(u_{2}, T\right)=0$. In this case we obtain

$$
\lambda_{2}=4 \sin 2\left(\frac{\pi}{T+1}\right)>0=M\left(u_{2}, T\right),
$$

and $u_{2}$ is an oscillating eigenvector as well as $u_{3}, \ldots, u_{T}$.

Remark 2. We point out that for a problem of type (4) there always exists at least a positive eigenvalue with a positive corresponding eigenfunction, namely, the first eigenvalue (see, e.g. [3] or [1]). Thus, denoting by $\lambda_{1}([0, T+1])$ the first eigenvalue of Eq. (4), by using Theorem 2, we deduce that

$$
\frac{4}{(T+1)^{2}}<\lambda_{1}([0, T+1]) \leq 1 .
$$


Moreover, we point out that the left-hand side inequality in (11) is a discrete variant of the celebrated Faber-Krahn inequality which is valid in the continuous case (see, e.g. $[8,10,11])$, since in the particular case when $T=2$ a simple computation shows that $\lambda_{1}([0,3])=1$ (actually, in this case 1 is the only eigenvalue of the problem), and thus, the left-hand side of inequality (11) can be rewritten in the following way

$$
\frac{4}{(T+1)^{2}} \lambda_{1}([0,3])<\lambda_{1}([0, T+1]), \quad \forall T \geq 2 .
$$

Remark 3. We notice that by a simple computation it can be proved that in the degenerate case $T=1$ the only eigenvalue of problem (4) is $\lambda_{1}([0,2])=2$ while in the case $T=2$ the two eigenvalues of problem (4) are equal to 1 . Thus, under these conditions, we have the equality case in the right-hand side of inequality (5). In other words, the case when there is equality can occur.

We point out that with a similar proof the result of Theorem 2 can be extended to the following:

Theorem 3. Let $p>1$ be a fixed real number and let $a \geq 1$ and $b \geq a+2$ be two integers. Consider the problem

$$
\left\{\begin{array}{l}
-\Delta\left(|\Delta u(k-1)|^{p-2} \Delta u(k-1)\right)=\lambda|u(k)|^{p-2} u(k), \quad k \in[a, b-1], \\
u(a-1)=u(b)=0 .
\end{array}\right.
$$

Let $\lambda>0$ be an eigenvalue of problem (12) with the property that the corresponding eigenvector $u, u(k)>0$ for any $k \in[a, b-1]$. Then we have the estimates

$$
\frac{2^{p}}{(b-a+1)^{p}}<\lambda \leq \min \left\{1, \frac{1}{b-a} \cdot\left(1+\frac{\max \left\{u(1)^{p-1}, u(T)^{p-1}\right.}{\min \left\{u(1)^{p-1}, u(T)^{p-1}\right.}\right)\right\} .
$$

In the case when $p=2, a=1$ and $b=T+1$ in Theorem 3, we obtain Theorem 2 .

Finally, we recall that in the hypotheses of Theorem 3 the first eigenvalue, $\lambda_{1, p}([a-1, b])$, is defined from a variational point of view by the so-called Rayleigh quotient, that is

$$
\lambda_{1, p}([a-1, b])=\inf _{u \neq 0} \frac{\sum_{k=a}^{b}|\Delta u(k-1)|^{p}}{\sum_{k=a}^{b-1}|u(k)|^{p}} .
$$

We note that in the case $p=2$ we will use the notation $\lambda_{1}([a-1, b])$ instead of $\lambda_{1,2}([a-1, b])$. Theorem 3 shows that relation (11) can be extended thanks to the following relation

$$
\frac{4}{(b-a+1)^{2}}<\lambda_{1}([a-1, b]) \leq 1
$$




\section{Proof of Theorem 1}

- First, we show the existence of $\lambda_{0}>0$ such that any $\lambda \in\left(0, \lambda_{0}\right)$ is not an eigenvalue of problem (2).

Define the Rayleigh type quotient

$$
\lambda_{0}=\inf _{u \in H \backslash\{0\}} \frac{\sum_{k=1}^{T+1}|\Delta u(k-1)|^{2}+\sum_{k=1}^{T}|u(k)|^{q}}{\sum_{k=1}^{T} g(k)|u(k)|^{r}} .
$$

In the first instance, we prove that $\lambda_{0}>0$. In order to show that we start by pointing out that relations (14) and (11) imply

$$
\sum_{k=1}^{T+1}|\Delta u(k-1)|^{2} \geq \lambda_{1}([0, T+1]) \sum_{k=1}^{T}|u(k)|^{2} \geq \frac{4}{(T+1)^{2}} \sum_{k=1}^{T}|u(k)|^{2}, \quad \forall u \in H .
$$

Since, we have $2<r<q$ we deduce

$$
|u(k)|^{2}+|u(k)|^{q} \geq|u(k)|^{r}, \quad \forall u \in H, \quad \forall k \in[1, T] .
$$

Summing the above inequalities we obtain

$$
\sum_{k=1}^{T}|u(k)|^{2}+\sum_{k=1}^{T}|u(k)|^{q} \geq \sum_{k=1}^{T}|u(k)|^{r} \geq \frac{1}{|g|_{\infty}} \sum_{k=1}^{T} g(k)|u(k)|^{r}, \quad \forall u \in H .
$$

Combining relations (17) and (18), we infer

$$
\begin{aligned}
\sum_{k=1}^{T+1}|\Delta u(k-1)|^{2}+\sum_{k=1}^{T}|u(k)|^{q} & \geq \min \left\{\frac{4}{(T+1)^{2}}, 1\right\} \frac{1}{|g|_{\infty}} \sum_{k=1}^{T} g(k)|u(k)|^{r} \\
& =\frac{4}{(T+1)^{2}|g|_{\infty}} \sum_{k=1}^{T} g(k)|u(k)|^{r}, \quad \forall u \in H .
\end{aligned}
$$

The last inequality shows that

$$
\lambda_{0} \geq \frac{4}{(T+1)^{2}|g|_{\infty}}>0
$$

Let us now define, $J_{1}, I_{1}, J_{0}, I_{0}: H \rightarrow \mathbb{R}$ by

$$
J_{1}(u)=\frac{1}{2} \sum_{k=1}^{T+1}|\Delta u(k-1)|^{2}+\frac{1}{q} \sum_{k=1}^{T}|u(k)|^{q}, \quad I_{1}(u)=\frac{1}{r} \sum_{k=1}^{T} g(k)|u(k)|^{r},
$$

and

$$
J_{0}(u)=\sum_{k=1}^{T+1}|\Delta u(k-1)|^{2}+\sum_{k=1}^{T}|u(k)|^{q}, \quad I_{0}(u)=\sum_{k=1}^{T} g(k)|u(k)|^{r}
$$


Standard arguments imply that $J_{1}, I_{1} \in C^{1}(H, \mathbb{R})$ with

$$
\left\langle J_{1}^{\prime}(u), v\right\rangle=\sum_{k=1}^{T+1} \Delta u(k-1) \Delta v(k-1)+\sum_{k=1}^{T}|u(k)|^{q-2} u(k) v(k),
$$

and

$$
\left\langle I_{1}^{\prime}(u), v\right\rangle=\sum_{k=1}^{T} g(k)|u(k)|^{r-2} u(k) v(k)
$$

for any $u, v \in H$.

Lemma 1. Let $\lambda_{0}$ be defined by relation (16). Then no $\lambda \in\left(0, \lambda_{0}\right)$ is an eigenvalue of problem (2).

Proof. Indeed, assuming by contradiction that there exists $\lambda \in\left(0, \lambda_{0}\right)$ an eigenvalue of problem (2), it follows that we can find $w_{\lambda} \in H \backslash\{0\}$ such that

$$
\left\langle J_{1}^{\prime}\left(w_{\lambda}\right), v\right\rangle=\lambda\left\langle I_{1}^{\prime}\left(w_{\lambda}\right), v\right\rangle, \quad \forall v \in H
$$

Letting $v=w_{\lambda}$ we deduce $\left\langle J_{1}^{\prime}\left(w_{\lambda}\right), w_{\lambda}\right\rangle=\lambda\left\langle I_{1}^{\prime}\left(w_{\lambda}\right), w_{\lambda}\right\rangle$, or

$$
J_{0}\left(w_{\lambda}\right)=\lambda I_{0}\left(w_{\lambda}\right) .
$$

Since $w_{\lambda} \neq 0$ we have that $J_{0}\left(w_{\lambda}\right)>0$ and thus, $I_{0}\left(w_{\lambda}\right)>0$. Combining that fact with the ideas that $\lambda \in\left(0, \lambda_{0}\right)$ and $\lambda_{0}=\inf _{u \in H \backslash\{0\}} \frac{J_{0}(u)}{I_{0}(u)}$ we infer

$$
J_{0}\left(w_{\lambda}\right) \geq \lambda_{0} I_{0}\left(w_{\lambda}\right)>\lambda I_{0}\left(w_{\lambda}\right)=J_{0}\left(w_{\lambda}\right),
$$

which is a contradiction. The proof of Lemma 1 is complete.

- Secondly, we show that there exists $\lambda_{1}$ such that any $\lambda \in\left(\lambda_{1}, \infty\right)$ is an eigenvalue of problem (2).

For any $\lambda>0$ we define the functional $S_{\lambda}: H \rightarrow \mathbb{R}$ by

$$
S_{\lambda}(u)=J_{1}(u)-\lambda I_{1}(u), \quad \forall u \in H .
$$

We notice that $S_{\lambda} \in C^{1}(H, \mathbb{R})$ with the derivative given by

$$
\left\langle S_{\lambda}^{\prime}(u), v\right\rangle=\left\langle J_{1}^{\prime}(u), v\right\rangle-\lambda\left\langle I_{1}^{\prime}(u), v\right\rangle, \quad \forall u, v \in H .
$$

Thus, $\lambda$ is an eigenvalue of problem (2) if and only if there exists $u_{\lambda} \in H \backslash\{0\}$ a critical point of $S_{\lambda}$.

Lemma 2. For any $\lambda \in(0, \infty)$ the functional $S_{\lambda}$ is coercive, i.e. $\lim _{\|u\| \rightarrow \infty}$ $S_{\lambda}(u)=\infty$. 
Proof. It is obvious that

$$
S_{\lambda}(u) \geq \frac{1}{2}\|u\|^{2}+\frac{1}{q} \sum_{k=1}^{T}|u(k)|^{q}-\frac{|g|_{\infty}}{r} \sum_{k=1}^{T}|u(k)|^{r},
$$

for any $u \in H$. For any $m \geq 2$ let us denote

$$
|u|_{m}=\left(\sum_{k=1}^{T}|u(k)|^{m}\right)^{1 / m} .
$$

It is not difficult to notice that each $|\cdot|_{m}, m \geq 2$, is a norm on $H$. Since $H$ is a finite dimensional Hilbert space we deduce that for any $m_{1}, m_{2} \geq 2$ the norms $|\cdot|_{m_{1}},|\cdot|_{m_{2}}$ and $\|\cdot\|$ are equivalent.

The above pieces of information imply that there exist two positive constants $C_{1}$ and $C_{2}$ such that

$$
S_{\lambda}(u) \geq \frac{1}{2}\|u\|^{2}+C_{1}\|u\|^{q}-C_{2}\|u\|^{r},
$$

for any $u \in H$. Since $2<r<q$, the proof of Lemma 2 is complete.

Define

$$
\lambda_{1}=\inf _{u \in H \backslash\{0\}} \frac{\frac{1}{2} \sum_{k=1}^{T+1}|\Delta u(k-1)|^{2}+\frac{1}{q} \sum_{k=1}^{T}|u(k)|^{q}}{\frac{1}{r} \sum_{k=1}^{T} g(k)|u(k)|^{r}} .
$$

Due to (16), a simple estimate shows that

$$
r \min \left\{\frac{1}{2}, \frac{1}{q}\right\} \lambda_{0} \leq \lambda_{1} \leq r \max \left\{\frac{1}{2}, \frac{1}{q}\right\} \lambda_{0} .
$$

Since $2<r<q$, we clearly have

$$
\frac{r}{q} \lambda_{0} \leq \lambda_{1} \leq \frac{r}{2} \lambda_{0}
$$

In particular, (20) and the left-hand size of (22) imply $\lambda_{1}>0$.

Lemma 3. Any $\lambda \in\left(\lambda_{1}, \infty\right)$ is an eigenvalue of problem (2).

Proof. We fix $\lambda \in\left(\lambda_{1}, \infty\right)$. By Lemma 2, we deduce that $S_{\lambda}$ is coercive. On the other hand, it is clear that the functional $S_{\lambda}$ is weakly lower semi-continuous. These two facts enable us to apply [13, Theorem 1.2] in order to prove that there exists $u_{\lambda} \in H$ a global minimum point of $S_{\lambda}$. 
Next, we show that $u_{\lambda}$ is not trivial. Indeed, since $\lambda_{1}=\inf _{u \in H, u \neq 0} \frac{J_{1}(u)}{I_{1}(u)}$ and $\lambda>\lambda_{1}$ it follows that there exists $v_{\lambda} \in H$ such that

$$
J_{1}\left(v_{\lambda}\right)<\lambda I_{1}\left(v_{\lambda}\right)
$$

or

$$
S_{\lambda}\left(v_{\lambda}\right)<0
$$

In particular, $\inf _{H} S_{\lambda}<0$, and we conclude that $u_{\lambda} \neq 0$.

Next, we show that $\lambda$ is an eigenvalue of problem (2). Let $v \in H$ fixed. The above property of $u_{\lambda}$ gives that

$$
\left.\frac{d}{d \epsilon} S_{\lambda}\left(u_{\lambda}+\epsilon v\right)\right|_{\epsilon=0}=0
$$

or

$$
\left\langle J_{1}^{\prime}\left(u_{\lambda}\right), v\right\rangle-\lambda\left\langle I_{1}^{\prime}\left(u_{\lambda}\right), v\right\rangle=0, \quad \forall v \in H
$$

that means $\lambda$ is an eigenvalue of problem (2). The proof of Lemma 3 is complete.

- Next, we show that $\lambda_{1}$ is also an eigenvalue of problem (2). In order to do that we first prove the following result.

Lemma 4. $\lim _{\|u\| \rightarrow 0} \frac{J_{0}(u)}{I_{0}(u)}=\lim _{\|u\| \rightarrow \infty} \frac{J_{0}(u)}{I_{0}(u)}=\infty$.

Proof. Considering again the norms, $|\cdot|_{m}, m \geq 2$, defined in Lemma 2 and recalling that they are equivalent with the norm $\|\cdot\|$ we find that there exist two positive constants $D_{1}$ and $D_{2}$ such that

$$
\frac{J_{0}(u)}{I_{0}(u)} \geq \frac{\|u\|^{2}+D_{1}\|u\|^{q}}{D_{2}\|u\|^{r}}, \quad \forall u \in H \backslash\{0\} .
$$

Now taking into account that $2<r<q$, the conclusion of Lemma 4 immediately holds.

Lemma 5. The real number $\lambda_{1}$, given by relation (21), is an eigenvalue of problem (2).

Proof. Let $\left(\lambda_{n}\right)$ be a sequence in $\mathbb{R}$ such that $\lambda_{n} \searrow \lambda_{1}$ as $n \rightarrow \infty$. By Lemma 3, we deduce that for each $n$ there exists $u_{n} \in H \backslash\{0\}$ such that

$$
\left\langle J_{1}^{\prime}\left(u_{n}\right), v\right\rangle-\lambda_{n}\left\langle I_{1}^{\prime}\left(u_{n}\right), v\right\rangle=0, \quad \forall v \in H .
$$

Taking $v=u_{n}$ in the above equality we find

$$
J_{0}\left(u_{n}\right)=\lambda_{n} I_{0}\left(u_{n}\right), \quad \forall n .
$$

The above equality and Lemma 4 imply that $\left(u_{n}\right)$ is a bounded sequence in $H$. Indeed, assuming by contradiction that $\left(u_{n}\right)$ is not bounded in $H$ it follows that 
passing eventually to a subsequence, still denoted by $\left(u_{n}\right)$ we have $\left\|u_{n}\right\| \rightarrow \infty$. On the other hand, the fact that $\lambda_{n} \searrow \lambda_{1}$ and relation (24) imply that for each $n$ large enough it holds true

$$
\frac{J_{0}\left(u_{n}\right)}{I_{0}\left(u_{n}\right)}=\lambda_{n} \leq \lambda_{1}+1 .
$$

Lemma 4 shows that the above inequality and the fact that $\left\|u_{n}\right\| \rightarrow \infty$ lead to a contradiction. Consequently, $\left(u_{n}\right)$ is bounded in $H$. We deduce the existence of $u \in H$ such that, passing eventually to a subsequence, $u_{n}$ converges to $u$ in $H$. Passing to the limit as $n \rightarrow \infty$ in (23) we get

$$
\left\langle J_{1}^{\prime}(u), v\right\rangle-\lambda_{1}\left\langle I_{1}^{\prime}(u), v\right\rangle=0, \quad \forall v \in H,
$$

i.e. $\lambda_{1}$ is an eigenvalue of problem (2) provided that $u \neq 0$.

Finally, we explain why $u \neq 0$. Assuming by contradiction that $u=0$ we deduce that $u_{n}$ converges to 0 in $H$. By relation (24) we deduce that for any $n$ the following equality holds

$$
\frac{J_{0}\left(u_{n}\right)}{I_{0}\left(u_{n}\right)}=\lambda_{n}
$$

Passing to the limit as $n \rightarrow \infty$ and taking into account the result of Lemma 4 and the fact that $\lambda_{n} \searrow \lambda_{1}$ we obtain a contradiction. The proof of Lemma 5 is complete.

- Finally, we point out that the conclusion of Theorem 1 holds true.

Proof of Theorem 1. In order to obtain the first part, it is enough to combine Lemmas 1, 3 and 5; in particular, we clearly have $\lambda_{0} \leq \lambda_{1}$. The first two inequalities of (3) come from (22) and (20), respectively.

It remains to prove the right-hand side inequality of (3), i.e. $\lambda_{1} \leq A$, where we use the notation $A=\frac{r(q-2)}{(q-r) \sum_{k=1}^{T} g(k)}\left(\frac{T(q-r)}{q(r-2)}\right)^{\frac{r-2}{q-2}}$. Fix $\tilde{u} \in H \backslash\{0\}$ by $\tilde{u}(k)=s>$ $0, k \in[1, T]$. Due to $(21)$, we have

$$
\lambda_{1} \leq \frac{\frac{1}{2} \sum_{k=1}^{T+1}|\Delta \tilde{u}(k-1)|^{2}+\frac{1}{q} \sum_{k=1}^{T}|\tilde{u}(k)|^{q}}{\frac{1}{r} \sum_{k=1}^{T} g(k)|\tilde{u}(k)|^{r}}=\frac{r\left(s^{2}+\frac{T}{q} s^{q}\right)}{s^{r} \sum_{k=1}^{T} g(k)} .
$$

Taking the function $h:(0, \infty) \rightarrow(0, \infty)$ defined by

$$
h(s)=\frac{r\left(s^{2}+\frac{T}{q} s^{q}\right)}{s^{r} \sum_{k=1}^{T} g(k)}
$$

one can easily show that its minimum is attained at the point $s_{0}=\left(\frac{q(r-2)}{T(q-r)}\right)^{\frac{1}{q-2}}$, the minimum value being $h\left(s_{0}\right)=A$. This concludes the proof. 
Remark 4. We notice that the spectrum of problem (2) is not completely described by our paper. Although we have estimates for $\lambda_{0}$ and $\lambda_{1}$, at this stage we are not able to say if $\lambda_{0}=\lambda_{1}$ or $\lambda_{0}<\lambda_{1}$. Note that $\lambda_{0}$ and $\lambda_{1}$ are very close to each other whenever $r$ is close to 2 ; in that sense, see the first inequality in (3). Due to the nonhomogeneous nature of the problem (2), we are strongly convinced that we usually have $\lambda_{0}<\lambda_{1}$, i.e. there is a gap between $\lambda_{0}$ and $\lambda_{1}$. If so, the problem of the existence/nonexistence of eigenvalues in the interval $\left[\lambda_{0}, \lambda_{1}\right)$ should be elucidated. This problem will hopefully be considered in a forthcoming paper.

\section{Acknowledgments}

The research of A. Kristály was supported by the János Bolyai Research Scholarship of the Hungarian Academy of Sciences and by Grant CNCSIS PCCE-55/2008 "Sisteme diferentiale in analiza neliniara si aplicatii". M. Mihăilescu has been supported by the grant CNCSIS TE-4/2010 "Proprietati calitative ale ecuatiilor cu derivate partiale si ale aproximarilor lor numerice". V. Rădulescu acknowledges the support through Grant CNCSIS PCCE-55/2008 "Sisteme diferentiale in analiza neliniara si aplicatii". S. Tersian is supported by Grant VU-MI-02/05. He is thankful to the Department of Mathematics and its Applications, CEU, Budapest, where part of this work was prepared during his visit.

\section{References}

[1] R. P. Agarwal, Difference Equations and Inequalities (Marcel Dekker Inc., 2000).

[2] R. P. Agarwal, K. Perera and D. O'Regan, Multiple positive solutions of singular and nonsingular discrete problems via variational methods, Nonlinear Anal. 58 (2004) 69-73.

[3] R. P. Agarwal, K. Perera and D. O'Regan, Multiple positive solutions of singular discrete $p$-Laplacian problems via variational methods, Adv. Difference Equ. 2005(2) (2005) 93-99.

[4] C. Bereanu, Topological degree methods for some nonlinear problems, Ph.D. Thesis, Université Catholique de Louvain (2006).

[5] H. Brezis, Analyse Fonctionnelle. Théorie et Applications, Collection Mathématiques Appliquées pour la Maîtrise (Masson, Paris, 1992).

[6] A. Cabada, A. Iannizzotto and S. Tersian, Multiple solutions for discrete boundary value problems, J. Math. Anal. Appl. 356 (2009) 418-428.

[7] X. Cai and J. Yu, Existence theorems for second-order discrete boundary value problems, J. Math. Anal. Appl. 320 (2006) 649-661.

[8] G. Faber, Beweis das unter allen homogenen Membranen von gleicher Fläche und gleicher Spannung die kreisförmige den tiefsten Grundton gibt, Münch. Ber. 1923 (1923) 169-172.

[9] W. G. Kelley and A. C. Peterson, Difference Equations. An Introduction with Applications (Harcourt Academic Press, 2001).

[10] E. Krahn, Über eine von Rayleigh formulierte Minimaleigenschaft des Kreises, Math. Ann. 94 (1925) 97-100.

[11] E. H. Lieb, On the lowest eigenvalue of the Laplacian for the intersection of two domains, Invent. Math. 74 (1983) 441-448. 
[12] M. Mihăilescu, V. Rădulescu and S. Tersian, Eigenvalue problems for anisotropic discrete boundary value problems, J. Difference Equ. Appl. 15 (2009) 557-567.

[13] M. Struwe, Variational Methods: Applications to Nonlinear Partial Differential Equations and Hamiltonian Systems (Springer, 1996).

[14] J. Yu and Z. Guo, On boundary value problems for a discrete generalized EmdenFowler equation, J. Math. Anal. Appl. 231 (2006) 18-31.

[15] G. Zhang and S. Liu, On a class of semipositone discrete boundary value problem, J. Math. Anal. Appl. 325 (2007) 175-182. 